\title{
Some properties for integro-differential operator defined by a fractional formal
}

\author{
Zainab E. Abdulnaby', Rabha W. Ibrahim² and Adem Kılıçman ${ }^{3^{*}}$
}

\author{
*Correspondence: \\ akilicman@yahoo.com \\ ${ }^{3}$ Department \\ of Mathematics, Institute \\ for Mathematical Research, \\ Universiti Putra Malaysia \\ (UPM), 43400 Serdang, \\ Selangor, Malaysia \\ Full list of author information \\ is available at the end of the \\ article
}

\begin{abstract}
Recently, the study of the fractional formal (operators, polynomials and classes of special functions) has been increased. This study not only in mathematics but extended to another topics. In this effort, we investigate a generalized integro-differential operator $\mathfrak{J}_{m}(z)$ defined by a fractional formal (fractional differential operator) and study some its geometric properties by employing it in new subclasses of analytic univalent functions.
\end{abstract}

Keywords: Fractional calculus, Unit disk, Analytic functions, Integral operator, Univalent functions, Fractional differential operator

\section{Background}

The subject of fractional calculus (integral and derivative of any arbitrary real or complex order) has acquired significant popularity and major attention from several authors in various science due mainly to its direct involvement in the problems of differential equations in mathematics, physics, engineering and others for example Baskonus and Bulut (2015), Yin et al. (2015) and Bulut (2016). The fractional calculus has gained an interesting area in mathematical research and generalization of the (derivative and integral) operators and its useful utility to express the mathematical problems which often leads to problems to be solved see Yao et al. (2015), Baskonus (2016) and Kumar et al. (2016). Specifically, it utilized to define new classes and generalized many geometric properties and inequalities in complex domain. In another words, these operators are play an important role in geometric function theory to define new generalized subclasses of analytic univalent and then study their properties. By using the technique of convolution or Hadamard product, Sălăgean (1981) defined the differential operator $\mathcal{D}_{n}$ of the class of analytic functions and it is well known as Sălăgean operator. Followed by Al-Oboudi differential operator see Al-Oboudi (2004). Several authors have used the Š̆ lăgean operator to define and consider the properties of certain known and new classes of analytic univalent functions. We refer here some of them in recent years. Najafzadeh (2010) investigated a new subclass of analytic univalent functions with negative and fixed finitely coefficient based on Sălăgean and Ruscheweyh differential operators. Aouf et al. (2012) gave some results for certain subclasses of analytic functions based on the definition for Sălăgean operator with varying arguments. El-Ashwah (2014) used Sălăgean operator to define a new subclass of analytic functions and derived some subordination 
results for this subclass in open unit disk. Breaz et al. (2008) investigated a new general integral operator for certain holomorphic functions based on the Sălăgean differential operator and studied some properties for this integral operator on some subclasses of univalent function. Also, Deniz et al. (2012) defined a new general integral operator by considering the Hadamard product and gave new sufficient conditions for this operator to be univalent in $\mathbb{U}$. Breaz et al. (2014) defined two general integral operators $F_{\lambda}(z)$ and $G_{\lambda}(z)$ and investigated some geometric properties for these operators on subclasses of analytic function in open unit disk.

In this paper, we define a generalized mixed integro-differential operator $\mathfrak{J}_{m}(z)$ based on the concept of Breaz integral operator as well as the fractional differential operator and study some their geometric properties on some new subclasses in open unit disk.

\section{Preliminaries}

Let $\mathcal{A}$ denote the class of all functions of the form

$$
f(z)=z+\sum_{n=2}^{\infty} a_{n} z^{n}
$$

which are analytic function in the open unit disk $\mathbb{U}=\{z:|z|<1\}$ and usually normalized by $f(0)=f^{\prime}(0)-1=0$. Also, let $\mathcal{S}$ be the subclass of $\mathcal{A}$ consisting of functions $f$ of form (1) which are univalent in $\mathbb{U}$. We denote by $\mathcal{S}^{*}(\beta)$ and $\mathcal{K}(\beta), 0 \leq \beta<1$, the classes of starlike function and convex function in $\mathbb{U}$, respectively. For $f \in \mathcal{A}$, Esa et al. (2016a) introduced the following differential operator $\mathcal{T}^{\alpha, \delta}: \mathcal{A} \rightarrow \mathcal{A}$,

$$
\mathcal{T}^{\alpha, \delta} f(z):=z+\sum_{n=2}^{\infty} \frac{\Gamma(\delta+1)}{\Gamma(\alpha+1)} \frac{\Gamma(n+\alpha)}{\Gamma(n+\delta)} a_{n} z^{n} \quad(z \in \mathbb{U}),
$$

for some $(0<\alpha \leq 1),(0<\delta \leq 1)$ and $n \in \mathbb{N} \backslash\{0,1\}$. If $\alpha=\delta$ in (2), then we get

$$
\mathcal{T}_{z}^{\alpha, \delta} f(z)=f(z) \quad(z \in \mathbb{U})
$$

for more details see Esa et al. (2016b). Now, let define a new fractional differential operator $\mathcal{D}_{\lambda}^{k}: \mathcal{A} \rightarrow \mathcal{A}$ as follows

$$
\begin{aligned}
& \mathcal{D}_{\lambda}^{0} f(z)=f(z) \\
& \mathcal{D}_{\lambda}^{1} f(z)=(1-\lambda) \mathcal{T}^{\alpha, \delta} f(z)+\lambda z\left(\mathcal{T}^{\alpha, \delta} f(z)\right)^{\prime}, \\
& \mathcal{D}_{\lambda}^{k} f(z)=\mathcal{D}_{\lambda}\left(\mathcal{D}_{\lambda}^{k-1} f(z)\right) \quad(k \in \mathbb{N}, z \in \mathbb{U}) .
\end{aligned}
$$

In general, we write

$$
D_{\lambda}^{k} f(z)=z+\sum_{n=2}^{\infty} \Phi_{n, k}(\alpha, \delta, \lambda) a_{n} z^{n} \quad k \in=\{0,1,2, \ldots\}
$$

where

$$
\Phi_{n, k}(\alpha, \delta, \lambda)=\left[\frac{\Gamma(\delta+1)}{\Gamma(\alpha+1)} \frac{\Gamma(n+\alpha)}{\Gamma(n+\delta)}(1+(n-1) \lambda)\right]^{k} .
$$


When $\alpha=\delta, \lambda=1$ and $k=1$, we get Sălăgean operator see Sălăgean (1981) and when $\alpha=\delta$, we have Al-Oboudi differential operator see Al-Oboudi (2004). Afterwards, we introduce some new subclasses of $\mathcal{A}$ as follows.

Let $\mathcal{S}^{k}(\lambda, \phi)$ denote the class of functions $f \in \mathcal{A}$ which satisfies the following condition:

$$
\mathfrak{R}\left\{\frac{z \mathcal{D}_{\lambda}^{k+1} f(z)}{\mathcal{D}_{\lambda}^{k} f(z)}\right\}>\phi \quad(z \in \mathbb{U})
$$

for some $0 \leq \phi<1, \lambda \geq 0$ and $k \in\{0,1, \ldots\}$. Let $\mathcal{K}^{k}(\lambda, \phi)$ denote the class of functions $f \in \mathcal{A}$ which satisfies the following condition

$$
\mathfrak{R}\left\{1+\frac{z \mathcal{D}_{\lambda}^{k+2} f(z)}{\mathcal{D}_{\lambda}^{k+1} f(z)}\right\}>\phi \quad(z \in \mathbb{U})
$$

for some $0 \leq \phi<1, \lambda \geq 0$ and $k \in\{0,1, \ldots\}$. It is clear that, when $k=0$ in (4) and (5), then we have the well known function classes

$$
\mathcal{S}^{0}(\lambda, \phi)=\mathcal{S}^{*}(\phi) \text { and } \mathcal{K}^{0}(\lambda, \phi)=\mathcal{K}(\phi) .
$$

Further, let $\mathcal{N}^{k}(\lambda, \psi)$ the subclass of $\mathcal{A}$, consisting of the functions $f$, which satisfies the following

$$
\mathfrak{R}\left\{1+\frac{z \mathcal{D}_{\lambda}^{k+2} f(z)}{\mathcal{D}_{\lambda}^{k+1} f(z)}\right\}<\psi \quad(z \in \mathbb{U})
$$

and let $\mathcal{M}^{k}(\lambda, \psi)$ be subclass of $\mathcal{A}$ consisting of the functions $f$ which satisfies the following

$$
\mathfrak{R}\left\{\frac{z \mathcal{D}_{\lambda}^{k+1} f(z)}{\mathcal{D}_{\lambda}^{k} f(z)}\right\}<\psi \quad(z \in \mathbb{U})
$$

for some $\psi>1, \lambda \geq 0$ and $k \in\{0,1, \ldots\}$. It is obvious that, when $k=0$ in (6) and (7), then we obtain the following classes

$$
\mathcal{M}^{0}(\lambda, \psi)=\mathcal{M}(\psi) \text { and } \mathcal{N}^{0}(\lambda, \psi)=\mathcal{N}(\psi)
$$

were interested by Owa and Srivastava (2002), Dixit and Chandra (2008) and recently studied by Porwal (2011). Let a function $f$ is said to be in the class $\mathcal{K}^{k} \mathcal{L}(\rho, \varphi)$, if

$$
\mathfrak{R}\left\{1+\frac{z \mathcal{D}_{\lambda}^{k+2} f(z)}{\mathcal{D}_{\lambda}^{k+1} f(z)}\right\} \geq \rho\left|\frac{z \mathcal{D}_{\lambda}^{k+2} f(z)}{\mathcal{D}_{\lambda}^{k+1} f(z)}\right|+\varphi \quad(0 \leq \varphi<1)
$$

for some $\rho, \lambda \geq 0$ and for all $z \in \mathbb{U}$. When $k=0$ in (8), we have the function class studied in Shams and Kulkarni (2004). For $f_{j}, g_{j} \in \mathcal{A}$ and $v_{j}, \beta_{j}$ be positive real numbers, $j=\{1,2, \ldots, m\}$, we define the integral operator $\mathfrak{J}_{m}(z): \mathcal{A}^{m} \rightarrow \mathcal{A}$ by 


$$
\mathfrak{J}_{m}(z)=\int_{0}^{z} \prod_{j=1}^{m}\left(\frac{\mathcal{D}_{\lambda}^{k} f_{j}(t)}{t}\right)^{\beta_{j}}\left(\mathcal{D}_{\lambda}^{k+1} g_{j}(t)\right)^{v_{j}} d t
$$

Note that, this integral operator is generalization of the integral operator recently defined by Stanciu and Breaz (2014). Also, the integral operator $\mathfrak{J}_{m}(z)$ is generalizes the following operators defined and investigated by several researchers:

Remark 1 For $k=0$ and $f_{j}^{\prime}=g_{j}^{\prime}, j=\{1,2, \ldots, m\}$, we have integral operator defined as follows:

$$
\mathcal{F}_{v, \beta} f(z)=\int_{0}^{z} \prod_{j=1}^{m}\left(\frac{f_{j}(t)}{t}\right)^{\beta_{j}}\left(f_{j}^{\prime}(t)\right)^{v_{j}} d t
$$

studied and considered by Frasin (2011).

Remark 2 For $k=0$ and $v_{j}=0, j=\{1,2, \ldots, m\}$, we have the following integral operator

$$
\mathcal{I}_{m}(z)=\int_{0}^{z} \prod_{j=1}^{m}\left(\frac{f_{j}(t)}{t}\right)^{\beta_{j}} d t
$$

was considered by Breaz and Breaz (2002).

Remark 3 For $k=0$, and $\beta_{j}=0, j=\{1,2, \ldots, m\}$, we have the integral operator

$$
\mathcal{I}_{v_{m}}(z)=\int_{0}^{z} \prod_{j=1}^{m}\left(g_{j}^{\prime}(t)\right)^{v_{j}} d t
$$

which studied by Breaz et al. (2009). In particular, for $m=1, v_{1}=v, \beta_{1}=0$ and $g_{1}=g$, we have the integral operator

$$
\mathcal{I}_{v} f(z)=\int_{0}^{z}\left(g^{\prime}(t)\right)^{v} d t
$$

which was considered by Pascu and Pescar (1990).

Remark 4 For $k=0, m=1, v_{1}=0, \beta_{1}=\beta$ and $f_{1}=f$, we have the following operator

$$
\mathcal{I}_{\beta} f(z)=\int_{0}^{z}\left(\frac{f(t)}{t}\right)^{\beta} d t
$$


investigated by Miller et al. (1978). In particular, for $\beta=1$, we have the Alexander's integral operator

$$
\mathcal{I} f(z)=\int_{0}^{z} \frac{f(t)}{t} d t
$$

which was studied by Alexander (1915).

\section{Main results}

We start our first result.

Theorem 1 Let $v_{j}, \beta_{j}$ be positive real numbers, $j=\{1,2, \ldots, m\}$. If $f_{j} \in \mathcal{M}^{k}\left(\lambda, \psi_{j}\right), \psi_{j}>1$ and $g_{j} \in \mathcal{N}^{k}\left(\lambda, \eta_{j}\right), \eta_{j}>1, j=\{1,2, \ldots, m\}$, then the integral operator $\mathfrak{J}_{m}(z)$ given by (9) is in the class $\mathcal{N}^{k}(\lambda, \rho)$, where

$$
\rho=1+\sum_{j=1}^{m}\left[\beta_{j}\left(\psi_{j}-1\right)+v_{j}\left(\eta_{j}-1\right)\right]
$$

Proof On successive differentiation of $\mathfrak{J}_{m}(z)$ defined in (9), we obtain

$$
\mathfrak{J}_{m}^{\prime}(z)=\prod_{j=1}^{m}\left(\left(\frac{\mathcal{D}_{\lambda}^{k} f_{j}(z)}{z}\right)^{\beta_{j}}\left(\mathcal{D}_{\lambda}^{k+1} g_{j}(z)\right)^{v_{j}}\right)
$$

and

$$
\begin{aligned}
\mathfrak{J}_{m}^{\prime \prime}(z)= & \sum_{j=1}^{m}\left[\beta_{j}\left(\frac{\mathcal{D}_{\lambda}^{k} f_{j}(z)}{z}\right)^{\beta_{j}-1}\left(\frac{z \mathcal{D}_{\lambda}^{k+1} f_{j}(z)-\mathcal{D}_{\lambda}^{k} f_{j}(z)}{z^{2}}\right)\left(\mathcal{D}_{\lambda}^{k+1} g_{j}(z)\right)^{\nu_{j}}\right] \\
& \times \prod_{\substack{\ell=1 \\
\ell \neq j}}^{m}\left(\left(\frac{\mathcal{D}_{\lambda}^{k} f_{\ell}(z)}{z}\right)^{\beta_{\ell}}\left(\mathcal{D}_{\lambda}^{k+1} g_{j}(z)\right)^{\nu_{\ell}}\right)+\sum_{j=1}^{m}\left[\left(\frac{\mathcal{D}_{\lambda}^{k} f_{j}(z)}{z}\right)^{\beta_{j}} v_{j}\left(\mathcal{D}_{\lambda}^{k+1} g_{j}(z)\right)^{v_{j}-1}\right. \\
& \left.\times \mathcal{D}_{\lambda}^{k+2} g_{j}(z)\right] \prod_{\substack{\ell=1 \\
\ell \neq j}}^{m}\left(\left(\frac{\mathcal{D}_{\lambda}^{k} f_{\ell}(z)}{z}\right)^{\beta_{\ell}}\left(\mathcal{D}_{\lambda}^{k+1} g_{j}(z)\right)^{\nu_{\ell}}\right) \\
&
\end{aligned}
$$

By a calculation, we have

$$
\frac{z \mathfrak{J}_{m}^{\prime \prime}(z)}{\mathfrak{J}_{m}^{\prime}(z)}=\sum_{j=1}^{m}\left[\beta_{j}\left(\frac{z \mathcal{D}_{\lambda}^{k+1} f_{j}(z)}{\mathcal{D}_{\lambda}^{k} f_{j}(z)}-1\right)+v_{j} \frac{z \mathcal{D}_{\lambda}^{k+2} g_{j}(z)}{\mathcal{D}_{\lambda}^{k+1} g_{j}(z)}\right] .
$$

The Eq. (14) is equivalent to

$$
\frac{z \widetilde{J}_{m}^{\prime \prime}(z)}{\mathfrak{J}_{m}^{\prime}(z)}+1=\sum_{j=1}^{m}\left[\beta_{j}\left(\frac{z \mathcal{D}_{\lambda}^{k+1} f_{j}(z)}{\mathcal{D}_{\lambda}^{k} f_{j}(z)}-1\right)+v_{j} \frac{z \mathcal{D}_{\lambda}^{k+2} g_{j}(z)}{\mathcal{D}_{\lambda}^{k+1} g_{j}(z)}\right]+1 .
$$


By calculating the real part of both expressions in (15), we have

$$
\begin{aligned}
\mathfrak{R}\left\{\frac{z \mathfrak{J}_{m}^{\prime \prime}(z)}{\mathfrak{J}_{m}^{\prime}(z)}+1\right\} & =\sum_{j=1}^{m}\left[\beta_{j} \Re \frac{z \mathcal{D}_{\lambda}^{k+1} f_{j}(z)}{\mathcal{D}_{\lambda}^{k} f_{j}(z)}-\beta_{j}+v_{j} \mathfrak{R} \frac{z \mathcal{D}_{\lambda}^{k+2} g_{j}(z)}{\mathcal{D}_{\lambda}^{k+1} g_{j}(z)}\right]+1, \\
& =\sum_{j=1}^{m}\left[\beta_{j} \Re \frac{z \mathcal{D}_{\lambda}^{k+1} f_{j}(z)}{\mathcal{D}_{\lambda}^{k} f_{j}(z)}-\beta_{j}+v_{j} \Re\left(\frac{z \mathcal{D}_{\lambda}^{k+2} g_{j}(z)}{\mathcal{D}_{\lambda}^{k+1} g_{j}(z)}+1\right)-v_{j}\right]+1 .
\end{aligned}
$$

Since $f_{j} \in \mathcal{M}^{k}\left(\lambda, \psi_{j}\right), \psi_{j}>1$ and $g_{j} \in \mathcal{N}^{k}\left(\lambda, \eta_{j}\right), \eta_{j}>1, j=\{1,2, \ldots, m\}$, we have

$$
\begin{aligned}
\mathfrak{R}\left\{\frac{z \mathfrak{J}_{m}^{\prime \prime}(z)}{\mathfrak{J}_{m}^{\prime}(z)}+1\right\} & <\sum_{j=1}^{m}\left[\beta_{j} \psi_{j}-\beta_{j}+v_{j} \eta_{j}-v_{j}\right]+1 \\
& <\sum_{j=1}^{m}\left[\beta_{j}\left(\psi_{j}-1\right)+v_{j}\left(\eta_{j}-1\right)\right]+1
\end{aligned}
$$

Therefore, $\mathfrak{J}_{m}(z) \in \mathcal{N}^{k}(\lambda, \rho)$, where $\rho=1+\sum_{j=1}^{m}\left[\beta_{j}\left(\psi_{j}-1\right)+v_{j}\left(\eta_{j}-1\right)\right]$.

Let $k=0, m=1$ in Theorem 1 , we have

Corollary 1 Let $\beta, v$ be positive real numbers. If $f \in \mathcal{M}(\psi), \psi>1$ and $g \in \mathcal{N}(\eta), \eta>1$, then

$$
\mathfrak{J}(z)=\int_{0}^{z}\left(\frac{f(t)}{t}\right)^{\beta}\left(g^{\prime}(t)\right)^{v} d t
$$

is in the class $\mathcal{N}(\rho)$, where $\rho=1+\beta(\psi-1)+v(\eta-1)$.

Theorem 2 Let $\beta_{j}, v_{j}$ be positive real numbers, $j=\{1,2, \ldots, m\}$. We assume that $f_{j}, j=\{1,2, \ldots, m\}$ are starlike functions by order $\frac{1}{\beta_{j}}$ and that is $f_{j} \in \mathcal{S}^{k}\left(\lambda, \frac{1}{\beta_{j}}\right)$ and $g_{j} \in \mathcal{K}^{k} \mathcal{L}\left(\rho_{j}, \eta_{j}\right), \rho_{j} \geq 1,0 \leq \eta_{j}<1, j=\{1,2, \ldots, m\}$. If

$$
\sum_{j=1}^{m}\left[v_{j}\left(1-\eta_{j}\right)+\beta_{j}\right]-m<1
$$

then $\mathfrak{J}_{m}(z)$ given by (9) is in the class $\mathcal{K}^{k}(\lambda, \omega)$ where

$$
\omega=1+m+\sum_{j=1}^{m}\left[v_{j}\left(\eta_{j}-1\right)-\beta_{j}\right]
$$

Proof By following same methods as in Theorem 1, we have

$$
\begin{aligned}
\frac{z \mathfrak{J}_{m}^{\prime \prime}(z)}{\mathfrak{J}_{m}^{\prime}(z)} & =\sum_{j=1}^{m}\left[\beta_{j}\left(\frac{z \mathcal{D}_{\lambda}^{k+1} f_{j}(z)}{\mathcal{D}_{\lambda}^{k} f_{j}(z)}-1\right)+v_{j} \frac{z \mathcal{D}_{\lambda}^{k+2} g_{j}(z)}{\mathcal{D}_{\lambda}^{k+1} g_{j}(z)}\right] \\
& =\sum_{j=1}^{m}\left[\beta_{j} \frac{z \mathcal{D}_{\lambda}^{k+1} f_{j}(z)}{\mathcal{D}_{\lambda}^{k} f_{j}(z)}-\beta_{j}+v_{j} \frac{z \mathcal{D}_{\lambda}^{k+2} g_{j}(z)}{\mathcal{D}_{\lambda}^{k+1} g_{j}(z)}\right],
\end{aligned}
$$


we can see that, (18) is equivalent to

$$
\frac{z \mathfrak{J}_{m}^{\prime \prime}(z)}{\mathfrak{J}_{m}^{\prime}(z)}+1=\sum_{j=1}^{m}\left[\beta_{j} \frac{z \mathcal{D}_{\lambda}^{k+1} f_{j}(z)}{\mathcal{D}_{\lambda}^{k} f_{j}(z)}-\beta_{j}+v_{j} \frac{z \mathcal{D}_{\lambda}^{k+2} g_{j}(z)}{\mathcal{D}_{\lambda}^{k+1} g_{j}(z)}\right]+1
$$

then by taking the real part of (19), we have

$$
\begin{aligned}
& \mathfrak{R}\left\{\frac{z \mathfrak{J}_{m}^{\prime \prime}(z)}{\mathfrak{J}_{m}^{\prime}(z)}+1\right\} \\
& \quad=\sum_{j=1}^{m}\left[\beta_{j} \Re \frac{z \mathcal{D}_{\lambda}^{k+1} f_{j}(z)}{\mathcal{D}_{\lambda}^{k} f_{j}(z)}-\beta_{j}+v_{j} \Re\left(\frac{z \mathcal{D}_{\lambda}^{k+2} g_{j}(z)}{\mathcal{D}_{\lambda}^{k+1} g_{j}(z)}+1\right)-v_{i}\right]+1,
\end{aligned}
$$

but $f_{j} \in \mathcal{S}^{k}\left(\lambda, \frac{1}{\beta_{j}}\right)$, that means $\mathfrak{R}\left\{\frac{\mathcal{D}_{\lambda}^{k+1} f_{j}(z)}{\mathcal{D}_{\lambda}^{k} f_{j}(z)}\right\}>\frac{1}{\beta_{j}}$ and $g_{j} \in \mathcal{K}^{k} \mathcal{L}\left(\rho_{j}, \eta_{j}\right), \rho_{j} \geq 0$ and $0 \leq \eta_{j}<1, j=\{1,2, \ldots, m\}$, from (20), we have

$$
\begin{aligned}
\mathfrak{R}\left\{\frac{z \mathfrak{J}_{m}^{\prime \prime}(z)}{\mathfrak{J}_{m}^{\prime}(z)}+1\right\} & >\sum_{j=1}^{m}\left[1-\beta_{j}+v_{j}\left(\rho_{i}\left|\frac{z \mathcal{D}_{\lambda}^{k+2} g_{j}(z)}{\mathcal{D}_{\lambda}^{k+1} g_{j}(z)}\right|+\eta_{j}\right)-v_{j}\right]+1 \\
& >1+m-\sum_{j=1}^{m} \beta_{j}+\sum_{j=1}^{m} v_{j} \rho_{i}\left|\frac{z \mathcal{D}_{\lambda}^{k+2} g_{j}(z)}{\mathcal{D}_{\lambda}^{k+1} g_{j}(z)}\right|+\sum_{j=1}^{m} v_{j}\left(\eta_{j}-1\right) .
\end{aligned}
$$

Since, $v_{j} \rho_{j}\left|\frac{z \mathcal{D}_{\lambda}^{k+2} g_{j}(z)}{\mathcal{D}_{\lambda}^{k+1} g_{j}(z)}\right|>0$, we have

$$
\begin{aligned}
\mathfrak{R}\left\{\frac{z \mathfrak{J}_{m}^{\prime \prime}(z)}{\mathfrak{J}_{m}^{\prime}(z)}+1\right\} & >1+m-\sum_{j=1}^{m} \beta_{j}+\sum_{j=1}^{m} v_{j}\left(\eta_{j}-1\right), \\
& >1+m+\sum_{j=1}^{m}\left[v_{j}\left(\eta_{j}-1\right)-\beta_{j}\right] .
\end{aligned}
$$

By using the condition in (17), we have that $\mathfrak{J}_{m}(z) \in \mathcal{K}^{k}(\lambda, \omega)$, where

$$
\omega=1+m+\sum_{j=1}^{m}\left[v_{j}\left(\eta_{j}-1\right)-\beta_{j}\right] .
$$

By setting $k=0$ and $m=1$ in Theorem 2, we have the following result.

Corollary 2 Let $\beta, v$ be positive real numbers, We assume that $f \in \mathcal{S}^{*}\left(\frac{1}{\beta}\right)$, $g \in \mathcal{K} \mathcal{L}(\rho, \eta), \rho \geq 0$ and $0 \leq \eta<1$. If

$$
\beta+v(1-\eta)<2
$$

then the integral operator

$$
\mathfrak{J}(z)=\int_{0}^{z}\left(\frac{f(t)}{t}\right)^{\beta}\left(g^{\prime}(z)\right)^{v} d t
$$

is in the class $\mathcal{K}(\omega)$ where $\omega=2+v(\eta-1)-\beta$. 


\section{Conclusion}

In geometric function theory, we defined and studied a new integro -differential operator $\mathfrak{J}_{m}(z)$, with a new classes of analytic and univalent functions. This operator is generalized and modified recent various fractional differential operators.

\section{Authors' contributions}

The authors, ZA, RI and AK contributed equally to the writing of this paper. All authors read and approved the final manuscript.

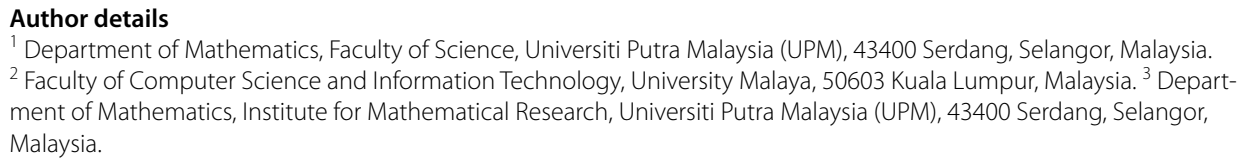

\section{Acknowledgements}

The authors would like to thank the referees for valuable suggestions and comments, which helped the authors to improve this article substantially.

\section{Competing interests}

The authors declare that they have no competing interests.

Received: 9 March 2016 Accepted: 12 June 2016

Published online: 27 June 2016

\section{References}

Al-Oboudi FM (2004) On univalent functions defined by a generalized Sălăgean operator. Int J Math Math Sci 2004(27):1429-1436

Alexander JW (1915) Functions which map the interior of the unit circle upon simple regions. Ann Math 17(1):12-22

Aouf MK, El-Ashwah RM, Hassan AAM, Hassan AH (2012) On subordination results for certain new classes of analytic functions defined by using Salagean operator. Bull Math Anal Appl 4(1):239-246

Baskonus HM (2016) Analytical and numerical methods for solving nonlinear partial differential equations-II. In: 1st international symposium on computational mathematics and engineering sciences, Errichidia/Morocco; 03-06 March 2016

Baskonus HM, Bulut H (2015) On the numerical solutions of some fractional ordinary differential equations by fractional Adams-Bashforth-Moulton method. Open Math 13(1):547-556

Breaz D, Breaz N (2002) Two integral operators. Stud Univ Babes-Bolyai Math 47(3):13-19

Breaz D, Owa S, Breaz N (2008) A new integral univalent operator. Acta Univ Apulensis Math Inform 16:11-16

Breaz D, Güney HO, Salagean GS (2009) A new general integral operator. Tamsui Oxf J Math Sci 25(4):407-414

Breaz D, Owa S, Breaz N (2014) Some properties for general integral operators. Adv Math 3(1):9-14

Bulut H (2016) Analytical and numerical methods for solving nonlinear partial differential equations-I. In: 1st international symposium on computational mathematics and engineering sciences, Errichidia/Morocco, 03-06 March 2016

Deniz E, Răducanu D, Orhan H (2012) On the univalence of an integral operator defined by Hadamard product. Appl Math Lett 25(2):179-184

Dixit KK, Chandra V (2008) On subclass of univalent functions with positive coefficients. Aligarh Bull Math 27(2):87-93

El-Ashwah RM (2014) Subordination results for certain subclass of analytic functions defined by Salagean operator. Acta Univ Apulensis 37:197-204

Esa Z, Kilicman A, Ibrahim RW, Ismail MR, Husain SS (2016a) Application of modified complex tremblay operator. In: Proceeding of 2nd international conference on mathematical sciences and statistics (ICMSS2016). 26-28 January, Kuala Lumpur, Malaysia, pp 26-28

Esa Z, Srivastava HM, Kilicman A, Ibrahim RW (2016b) A novel subclass of analytic functions specified by a family of fractional derivatives in the complex domain. Filomat. http://arxiv.org/pdf/1511.01581.pdf

Frasin BA (2011) Univalence criteria for general integral operator. Math Commun 16(1):115-124

Kumar S, Kumar A, Baleanu D (2016) Two analytical methods for time-fractional nonlinear coupled Boussinesq-Burger's equations arise in propagation of shallow water waves. Nonlinear Dyn 1-17

Miller SS, Mocanu P, Reade MO (1978) Starlike integral operators. Pac J Math 79(1):157-168

Najafzadeh S (2010) Application of Salagean and Ruscheweyh operators on univalent holomorphic functions with finitely many coefficients. Fract Calc Appl Anal 13(5):517-520

Owa S, Srivastava HM (2002) Some generalized convolution properties associated with certain subclasses of analytic functions. J Inequal Pure Appl Math 3(3):1-13

Pascu NN, Pescar V (1990) On the integral operators of Kim-Merkes and Pfaltzgraff. Mathematica (Cluj) 32(55):185-192

Porwal S (2011) Mapping properties of an integral operator. Acta Univ Apulensis 27:151-155

Shams S, Kulkarni SR, Jahangiri JM (2004) Classes of uniformly starlike and convex functions. Int J Math Math Sci 2004(55):2959-2961

Stanciu L, Breaz D (2014) Some properties of a general integral operator. Bull Iran Math Soc 40(6):1433-1439 
Sălăgean GS (1981) Subclasses of univalent functions, complex analysis-fifth Romanian-Finnish seminar, part 1 (Bucharest, 1981). Lect Notes Math 1013:362-372

Yao JJ, Kumar A, Kumar S (2015) A fractional model to describe the Brownian motion of particles and its analytical solution. Adv Mech Eng 7(12):1687814015618,874

Yin XB, Kumar S, Kumar D (2015) A modified homotopy analysis method for solution of fractional wave equations. Adv Mech Eng 7(12):1687814015620330

Submit your manuscript to a SpringerOpen ${ }^{\circ}$ journal and benefit from:

- Convenient online submission

- Rigorous peer review

- Immediate publication on acceptance

- Open access: articles freely available online

- High visibility within the field

- Retaining the copyright to your article

Submit your next manuscript at $\boldsymbol{\nabla}$ springeropen.com 